\title{
Fractional Fourier Transform for Ultrasonic Chirplet Signal Decomposition
}

\author{
Yufeng Lu, ${ }^{1}$ Alireza Kasaeifard, ${ }^{2}$ Erdal Oruklu, ${ }^{2}$ and Jafar Saniie ${ }^{2}$ \\ ${ }^{1}$ Department of Electrical and Computer Engineering, Bradley University, Peoria, IL 61625, USA \\ ${ }^{2}$ Department of Electrical and Computer Engineering, Illinois Institute of Technology, Chicago, IL 60616, USA
}

Correspondence should be addressed to Erdal Oruklu, erdal@ece.iit.edu

Received 7 April 2012; Accepted 31 May 2012

Academic Editor: Mario Kupnik

Copyright ( 2012 Yufeng Lu et al. This is an open access article distributed under the Creative Commons Attribution License, which permits unrestricted use, distribution, and reproduction in any medium, provided the original work is properly cited.

\begin{abstract}
A fractional fourier transform (FrFT) based chirplet signal decomposition (FrFT-CSD) algorithm is proposed to analyze ultrasonic signals for NDE applications. Particularly, this method is utilized to isolate dominant chirplet echoes for successive steps in signal decomposition and parameter estimation. FrFT rotates the signal with an optimal transform order. The search of optimal transform order is conducted by determining the highest kurtosis value of the signal in the transformed domain. A simulation study reveals the relationship among the kurtosis, the transform order of FrFT, and the chirp rate parameter in the simulated ultrasonic echoes. Benchmark and ultrasonic experimental data are used to evaluate the FrFT-CSD algorithm. Signal processing results show that FrFT-CSD not only reconstructs signal successfully, but also characterizes echoes and estimates echo parameters accurately. This study has a broad range of applications of importance in signal detection, estimation, and pattern recognition.
\end{abstract}

\section{Introduction}

In ultrasonic imaging applications, the ultrasonic signal always contains many interfering echoes due to the complex physical properties of the propagation path. The pattern of the signal is greatly dependent on irregular boundaries, and the size and random orientation of material microstructures. For material characterization and flaw detection applications, it becomes a challenging problem to unravel the desired information using direct measurement and conventional signal processing techniques. Consequently, signal processing methods capable of analyzing the nonstationary behavior of ultrasonic signals are highly desirable for signal analysis and characterization of propagation path.

Various methods such as short-time Fourier transform, Wigner-Ville distribution, discrete wavelet transform, discrete cosine transform, and chirplet transform have been utilized to examine signals in joint time-frequency domain and to reveal how frequency changes with time in those signals [1-8]. Nevertheless, it is still challenging to adaptively analyze a broad range of ultrasonic signal: narrowband or broadband; symmetric or skewed; nondispersive or dispersive.

Recently, there has been a growing attention to fractional Fourier transform (FrFT), a generalized Fourier transform with an additional parameter (i.e., transform order). It was first introduced in 1980, and subsequently closed-form FrFT was studied [8-11] for time-frequency analysis. FrFT is a power signal analysis tool. Consequently, it has been applied to different applications such as high-resolution SAR imaging, sonar signal processing, blind source separation, and beamforming in medical imaging [12-15]. Short term FrFT, component-optimized FrFT, and locally optimized FrFT have also been proposed for signal decomposition [16$18]$.

In practice, signal decomposition problem is essentially an optimization problem under different design criteria. The optimization can be achieved either locally or globally, depending on the complexity of the signal, accuracy of estimation, and affordability of computational load. Consequently, the results of signal decomposition are not unique due to different optimization strategies and signal models. For ultrasonic signal analysis, local responses from 
TABLE 1: Parameter estimation results of two slightly overlapped ultrasonic echoes.

\begin{tabular}{|c|c|c|c|c|c|c|}
\hline & $\tau$ (us) & $f_{c}(\mathrm{MHz})$ & $\beta$ & $\alpha_{1}(\mathrm{MHz})^{2}$ & $\alpha_{2}(\mathrm{MHz})^{2}$ & $\theta(\operatorname{Rad})$ \\
\hline \multicolumn{7}{|l|}{ Echo 1} \\
\hline Actual parameter & 2.5 & 7.0 & 1 & 20 & 35 & $\pi / 6$ \\
\hline Estimated parameter & 2.50 & 7.00 & 1.00 & 20.00 & 35.00 & 0.52 \\
\hline \multicolumn{7}{|l|}{ Echo 2} \\
\hline Actual parameter & 3.0 & 5 & 1 & 25 & 20 & 0 \\
\hline Estimated parameter & 3.00 & 5.00 & 1.00 & 25.00 & 19.98 & 0 \\
\hline
\end{tabular}

TABLE 2: Parameter estimation results of two moderately overlapped ultrasonic echoes.

\begin{tabular}{|c|c|c|c|c|c|c|}
\hline & $\tau$ (us) & $f_{c}(\mathrm{MHz})$ & $\beta$ & $\alpha_{1}(\mathrm{MHz})^{2}$ & $\alpha_{2}(\mathrm{MHz})^{2}$ & $\theta(\mathrm{Rad})$ \\
\hline \multicolumn{7}{|l|}{ Echo 1} \\
\hline Actual parameter & 2.7 & 7.0 & 1 & 20 & 35 & $\pi / 6$ \\
\hline Estimated parameter & 2.70 & 7.02 & 1.00 & 20.04 & 33.55 & 0.67 \\
\hline \multicolumn{7}{|l|}{ Echo 2} \\
\hline Actual parameter & 3.0 & 5 & 1 & 25 & 20 & 0 \\
\hline Estimated parameter & 3.00 & 5.00 & 1.00 & 24.87 & 20.38 & 0.01 \\
\hline
\end{tabular}

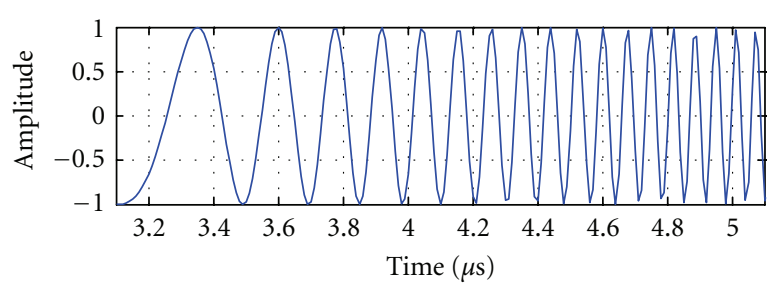

(a)

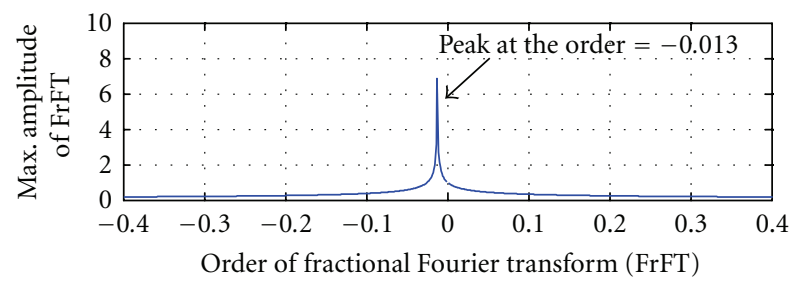

(b)

FIGURE 1: (a) A simulated LFM signal. (b) The optimal transform order tracked by Maximum amplitude of FrFT for different FrFT orders.

microstructure scattering and structural discontinuities are more of importance for detection and material characterization. Chirplet covers a board range of signals representing frequency-dependent scattering, attenuation and dispersion effects in ultrasonic testing applications. This study shows that FrFT has a unique property for processing chirptype echoes. Therefore, in this paper, the application of fractional Fourier transform for ultrasonic applications

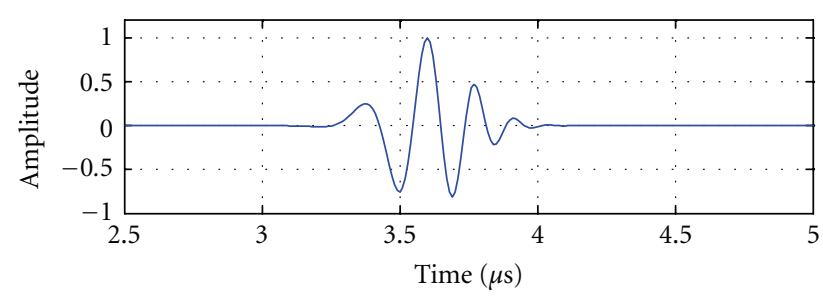

(a)

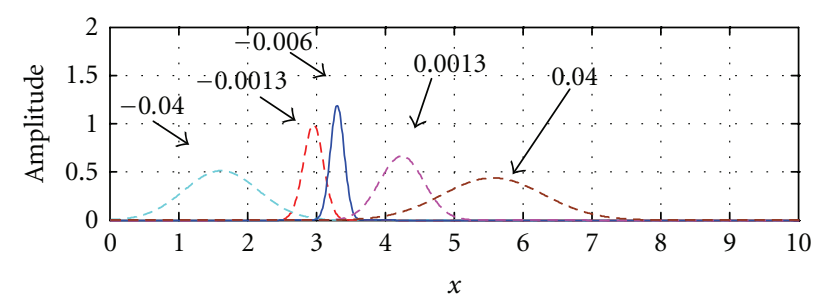

(b)

Figure 2: (a) a simulated ultrasonic single echo with $\Theta=$ [3.6 us $5 \mathrm{MHz} \quad 1 \quad 25 \mathrm{MHz}^{2} \quad 25 \mathrm{MHz}^{2}$ 0]. (b) Fractional Fourier transform of the signal in (a) for different transform orders.

has been explored. In particular, FrFT is introduced as a transformation tool for ultrasonic signal decomposition. FrFT is employed to estimate an optimal transform order, which corresponds to highest kurtosis value in the transform domain. The searching process of optimal transform order is based on a segmented signal for a local optimization. Then, the FrFT with the optimal transform order is applied to the entire signal in order to isolate the dominant echo 


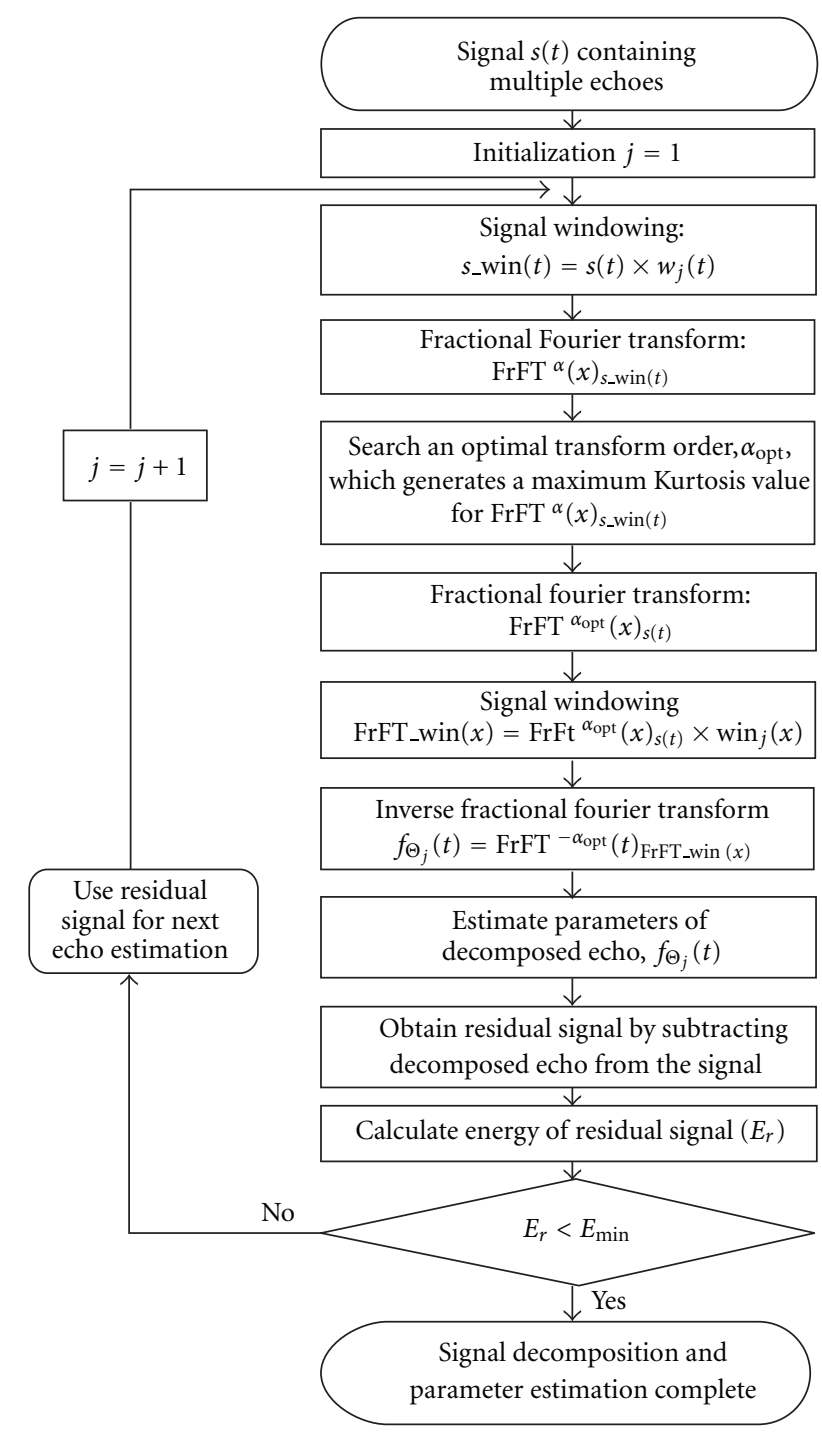

FIgURE 3: Flowchart of FrFT-CSD algorithm.

for parameter estimation. This echo isolation is applied iteratively to ultrasonic signal until a predefined stop criterion such as signal reconstruction error or the number of iterations is satisfied. Furthermore, each decomposed component is modeled using six-parameter chirplet echoes for a quantitative analysis of ultrasonic signals.

A bat signal is utilized as a benchmark to demonstrate the effectiveness of fractional Fourier transform chirplet signal decomposition (FrFT-CSD). To further evaluate the performance of FrFT-CSD, ultrasonic experimental data from different types of flaws such as flat bottom hole, sidedrilled hole and disk-type cracks are evaluated using FrFTCSD.

The outline of the paper is as follows. Section 2 reviews the properties of FrFT and the process of FrFT-based signal decomposition. Section 3 addresses how kurtosis, transformation order and chirp rate are related using simulated data. Section 4 presents the steps involved in FrFT-CSD

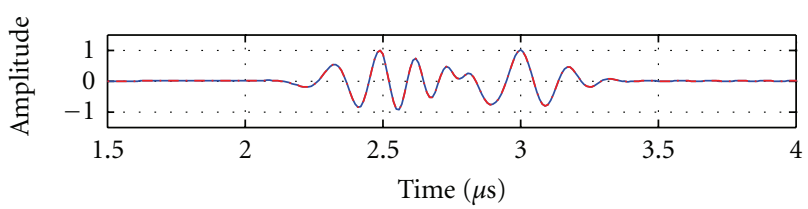

(a)

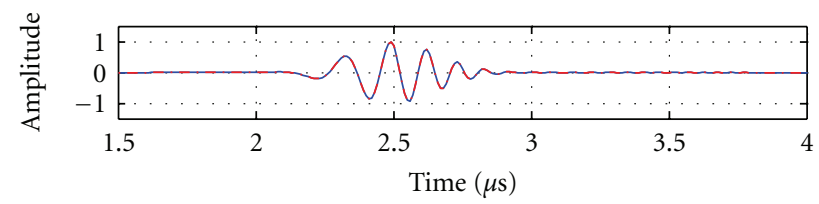

(b)

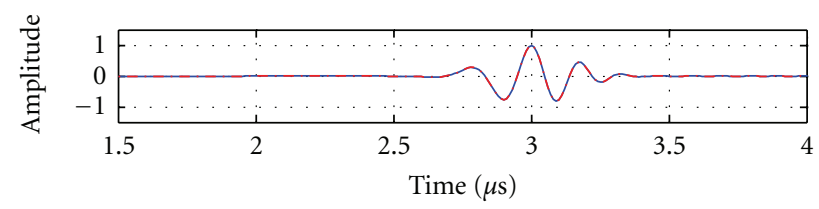

(c)

Figure 4: (a) Simulated ultrasonic echoes (20\% overlapped). (b) The first signal component. (c) The second signal component (simulated signal in blue, estimated signal in red).

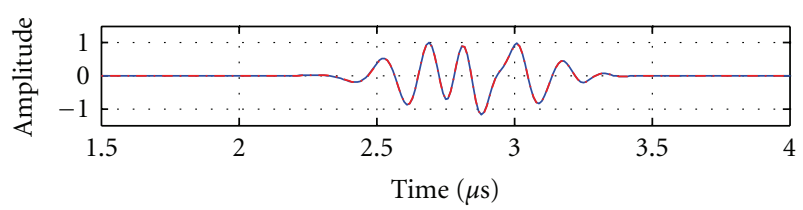

(a)

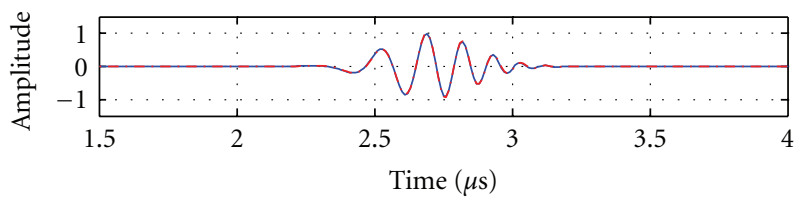

(b)

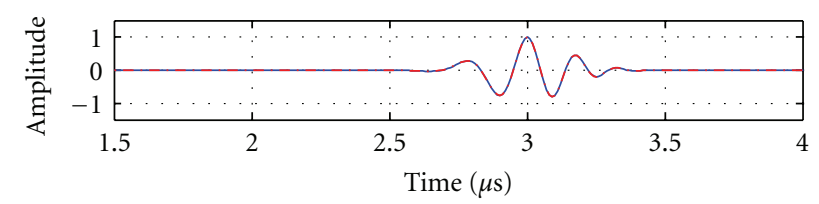

(c)

Figure 5: (a) Simulated ultrasonic echoes (50\% overlapped). (b) The first signal component. (c) The second signal component (simulated signal in blue, estimated signal in red).

algorithm. Section 5 performs a simulation study of FrFTCSD and parameter estimation for complex ultrasonic signals. Sections 5 and 6 show the results of a benchmark data (i.e., bat signal); the echo estimation results of benchmark data from side-drilled hole, and disk-shape cracks; the results of experimental data with high microstructure scattering echoes. 


\section{FrFT of Ultrasonic Chirp Echo}

FrFT of a signal, $f(t)$, is given by

$$
\begin{aligned}
F^{\alpha}(x)= & \frac{e^{-i((\pi / 4) \operatorname{sgn}(\pi \alpha / 2)-(\pi \alpha / 4))}}{(2 \pi|\sin (\pi \alpha / 2)|)^{1 / 2}} e^{(1 / 2) i x^{2} \cot (\pi \alpha / 2)} \\
& \times \int_{-\infty}^{\infty} e^{\left(-i(x t / \sin (\pi \alpha / 2))+(1 / 2) i t^{2} \cot (\pi \alpha / 2)\right)} f(t) d t,
\end{aligned}
$$

where $\alpha$ denotes transform order of FrFT and $x$ denotes the variable in transform domain.

It has been shown that if the transform order, $\alpha$, changes from 0 to 4 , (i.e., the rotation angle, $\phi$, changes from 0 to $2 \pi$ ), $F^{\alpha}(x)$ rotates the signal, $f(t)$, and projects it onto the line of angle, $\phi$, in time-frequency domain [19]. This property contributes to FrFT-based decomposition algorithm when applied to ultrasonic signals.
For ultrasonic applications, ultrasonic chirp echo is a type of signal often encountered in ultrasonic backscattered signals accounting for narrowband, broadband, and dispersive echoes. It can be modeled as [8]:

$$
\begin{gathered}
f_{\Theta}(t)=\beta \exp \left[-\alpha_{1}(t-\tau)^{2}+i 2 \pi f_{c}(t-\tau)\right. \\
\left.+i \alpha_{2}(t-\tau)^{2}+i \theta\right]
\end{gathered}
$$

where $\Theta=\left[\tau f_{c} \beta \alpha_{1} \alpha_{2} \theta\right]$ denotes the parameter vector, $\tau$ is the time-of-arrival, $f_{c}$ is the center frequency, $\beta$ is the amplitude, $\alpha_{1}$ is the bandwidth factor, $\alpha_{2}$ is the chirp-rate, and $\theta$ is the phase.

Hence, for the ultrasonic Gaussian chirp echo, $f_{\Theta}(t)$, the magnitude of $F^{\alpha}(x)$ given by (1) can be expressed as

$$
\begin{aligned}
\left|F^{\alpha}(x)\right| & =\frac{1}{(2 \pi|\sin (\pi \alpha / 2)|)^{1 / 2}}\left|\int_{-\infty}^{\infty} e^{\left(-i(x t / \sin (\pi \alpha / 2))+(1 / 2) i t^{2} \cot (\pi \alpha / 2)\right)} f(t) d t\right| \\
& =\frac{1}{\left(2 \sqrt{\alpha_{1}^{2}+\left(\alpha_{2}+(1 / 2) \cot (\pi \alpha / 2)\right)^{2}}|\sin (\pi \alpha / 2)|\right)^{1 / 2}}\left|e^{\left(B^{2}-4 A C\right) / 4 A}\right|,
\end{aligned}
$$

where the integration part can be written as

$$
\begin{aligned}
& \left|\int_{-\infty}^{\infty} e^{\left(-i(x t / \sin (\pi \alpha / 2))+(1 / 2) i t^{2} \cot (\pi \alpha / 2)\right)} f(t) d t\right| \\
& \quad=\left|\int_{-\infty}^{\infty} e^{-\left[t^{2}\left(\alpha_{1}-\alpha_{2} i-(1 / 2) i \cot (\pi \alpha / 2)\right)+t\left(2 \alpha_{2} \tau i-2 \alpha_{1} \tau-2 \pi f_{0} i+x i \csc (\pi \alpha / 2)\right)+\left(\alpha_{1} \tau^{2}-\alpha_{2} i \tau^{2}-\theta+2 \pi f_{0} \tau i\right)\right]} d t\right| \\
& \quad=\left|\sqrt{\frac{\pi}{A}} e^{\left(B^{2}-4 A C\right) / 4 A}\right|,
\end{aligned}
$$

with $A=\alpha_{1}-\alpha_{2} i-(1 / 2) i \cot (\pi \alpha / 2), B=2 \alpha_{2} \tau i-2 \alpha_{1} \tau-$ $2 \pi f_{0} i+x i \csc (\pi \alpha / 2)$, and $C=\alpha_{1} \tau^{2}-\alpha_{2} i \tau^{2}-\theta+2 \pi f_{0} \tau i$.

From (3), it can be seen that, for a linear frequency modulation (LFM) signal (i.e., $\alpha_{1}=0$ ), if the transformation order, $\alpha$, satisfies the following equation:

$$
\begin{aligned}
& \left(\alpha_{2}+\frac{1}{2} \cot \frac{\pi \alpha}{2}\right) \sin \frac{\pi \alpha}{2}=0, \\
& \alpha=-\frac{2}{\pi} \tan ^{-1}\left(\frac{1}{2 \alpha_{2}}\right),
\end{aligned}
$$

then the $\left|F^{\alpha}(x)\right|$ compacts to a delta function. This means that fractional Fourier transform can be used to compress the duration and compact the energy of ultrasonic chirp echo with an optimal transform order. Optimal transform order can be determined using kurtosis. The energy compaction is a desirable property for ultrasonic signal decomposition, which allows using a window in FrFT domain for isolation of an echo of interest.

\section{Kurtosis and FrFT Order}

Kurtosis is commonly used in statistics to evaluate the degree of peakedness for a distribution $[20,21]$. It is defined as the ratio of 4 th-order central moment and square of 2 nd-order central moment:

$$
K(\alpha)=\frac{\mu_{4}\left(F^{\alpha}(x)\right)}{\left[\mu_{2}\left(F^{\alpha}(x)\right)\right]^{2}},
$$

where $\mu_{4}(\bullet)$ denotes 4 th-order central moment and $\mu_{2}(\bullet)$ denotes 2nd-order central moment. A signal with high kurtosis means that it has a distinct peak around the mean. In the literatures of FrFT [18, 19, 22], kurtosis is typically used as a metric to search the optimal transform order of FrFT. Different transform order directs the degree of signal rotation caused by FrFT, and this rotation affects the extent of energy compaction of the transformed signal.

Figure 1(a) shows a chirp signal with the param-

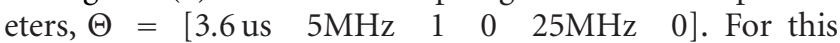




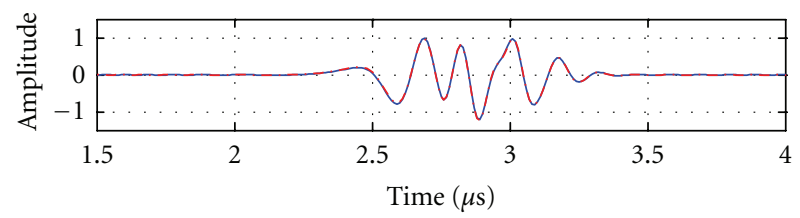

(a)

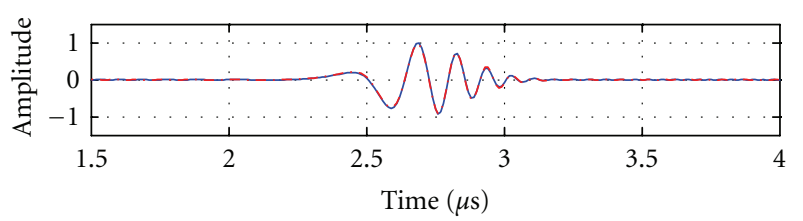

(b)

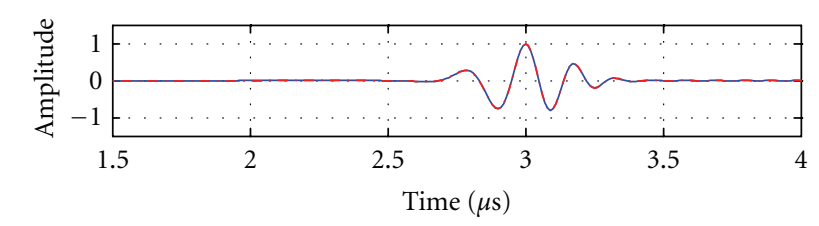

(c)

FIgURE 6: (a) Simulated ultrasonic echoes (70\% overlapped). (b) The first estimated echo component. (c) The second estimated echo component (simulated signal in blue, estimated signal in red).
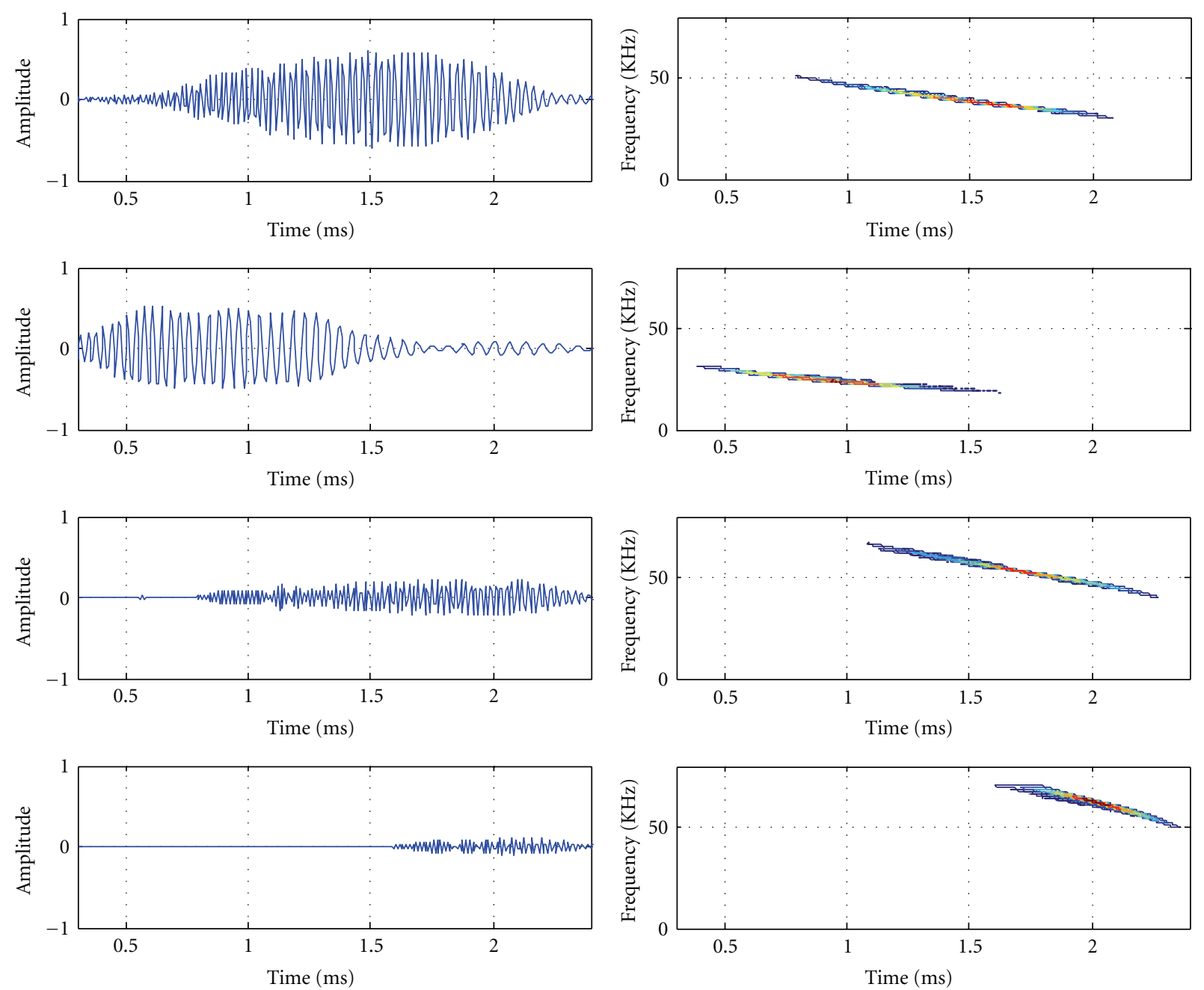

(a)

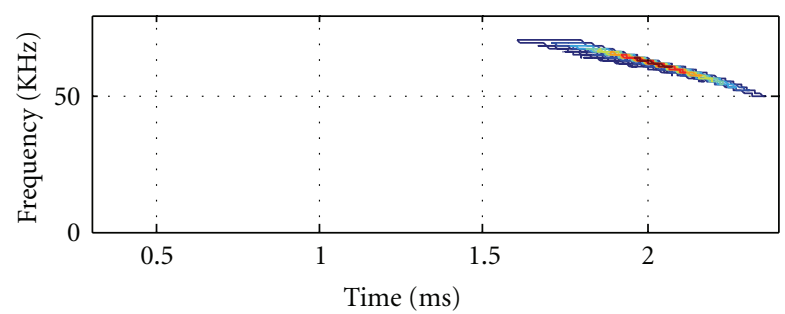

(b)

Figure 7: Left column (top to bottom): decomposed bat signal components in time domain. Right column (top to down): Wigner-Ville distribution of the corresponding signals in left column. 
TABLE 3: Parameter estimation results of two heavily overlapped ultrasonic echoes.

\begin{tabular}{lcccccc}
\hline & $\tau(\mathrm{us})$ & $f_{c}(\mathrm{MHz})$ & $\beta$ & $\alpha_{1}(\mathrm{MHz})^{2}$ & $\alpha_{2}(\mathrm{MHz})^{2}$ & $\theta(\mathrm{Rad})$ \\
\hline Echo 1 & & & & & & \\
$\quad$ Actual parameter & 2.7 & 6 & 1 & 20 & 55 & $\pi / 6$ \\
$\quad$ Estimated parameter & 2.70 & 6.11 & 0.97 & 18.87 & & 0.72 \\
\hline Echo 2 & 3.0 & 5 & 1 & 25 & 20 & 0 \\
$\quad$ Actual parameter & 3.00 & 5.00 & 1.00 & 25.14 & 20.38 & 0.01 \\
$\quad$ Estimated parameter & & & & & & \\
\hline
\end{tabular}

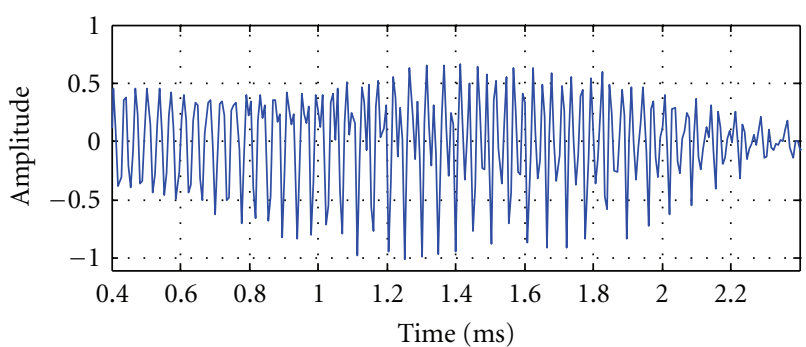

(a)

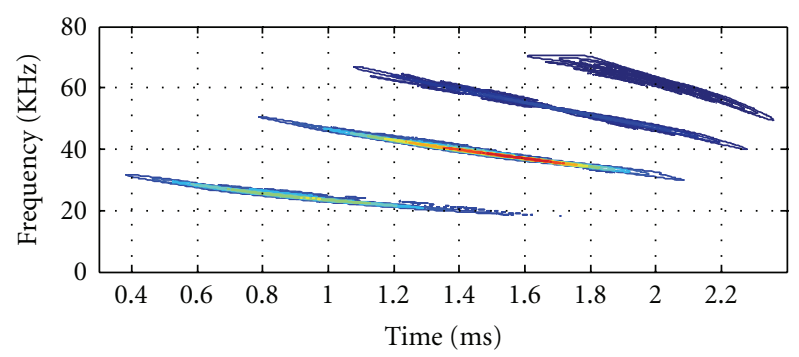

(b)

Figure 8: (a) Reconstructed bat signal. (b) Summed Wigner Ville distribution of the decomposed signals in (a).

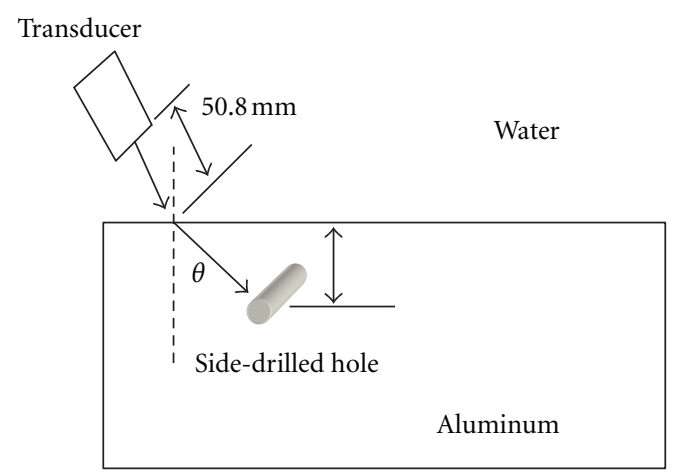

Figure 9: Experiment setup for SDH blocks.

example, the bandwidth factor equals to zero (see (2)), and according to (5), the optimal transform order can be calculated as

$$
\alpha=-\frac{2}{\pi} \tan ^{-1}\left(\frac{1}{2 \alpha_{2}}\right)=-0.013 .
$$

As shown in Figure 1(b), this optimal order can also be determined by direct search for the maximum amplitude

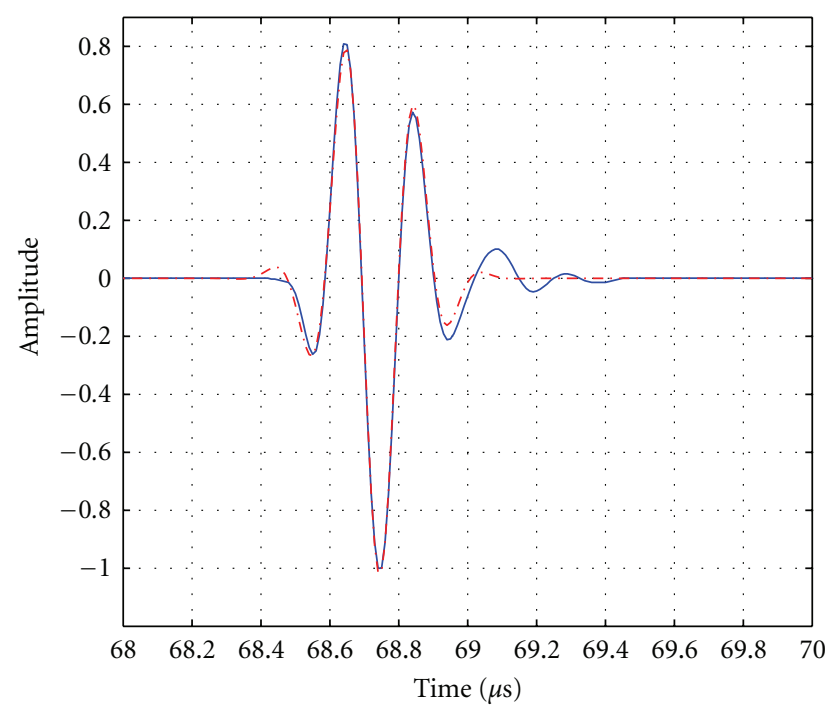

FIGURE 10: Ultrasonic data from the front surface superimposed with the estimated chirplet (depicted in dashed red color line).

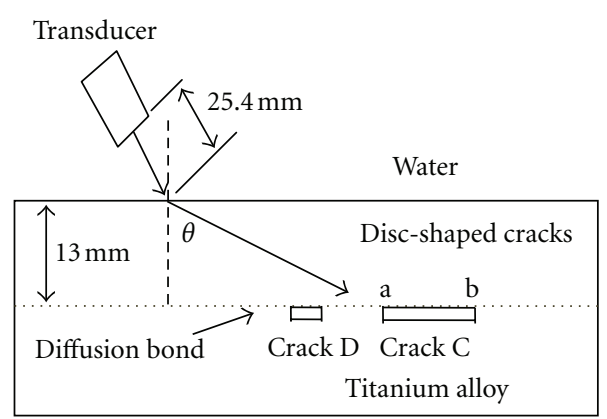

FIGURE 11: Experiment setup for disc-shaped cracks in a diffusionbonded titanium alloy.

of FrFT using different transform orders according to (3). The transform order corresponding to the maximum FrFT among all transform orders matches the theoretical result given in (7).

For ultrasonic applications, the chirp echo is bandlimited. For example, Figure 2(a) shows a band-limited single chirp echo with the parameters $\Theta=[3.6$ us $5 \mathrm{MHz}$ $125 \mathrm{MHz} \quad 25 \mathrm{MHz} \quad 0$ ]. Chirplet is a model widely used in ultrasonic NDE applications. Figure 2 illustrates the FrFT of a chirplet using different transform orders. In particular, 


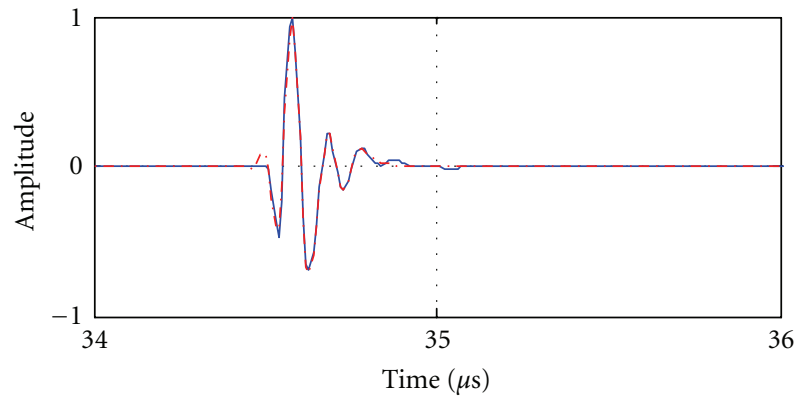

(a)

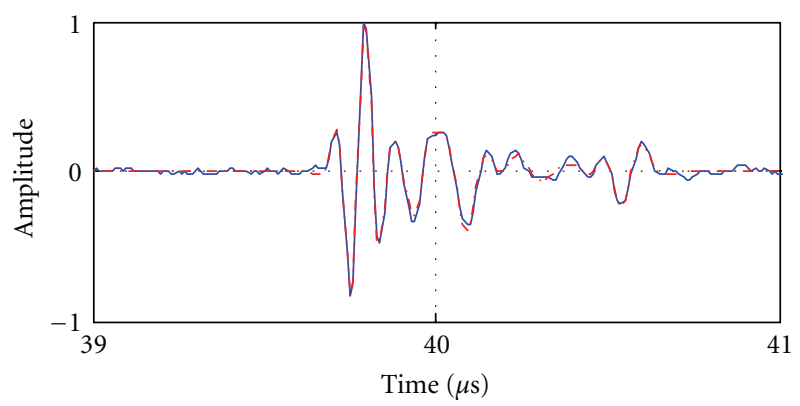

(c)

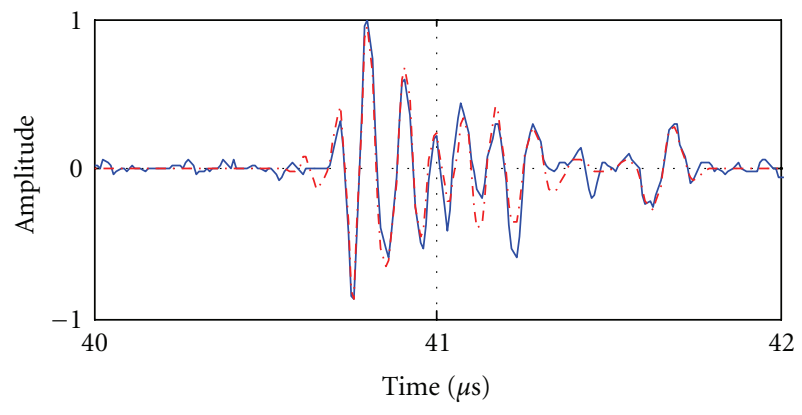

(e)

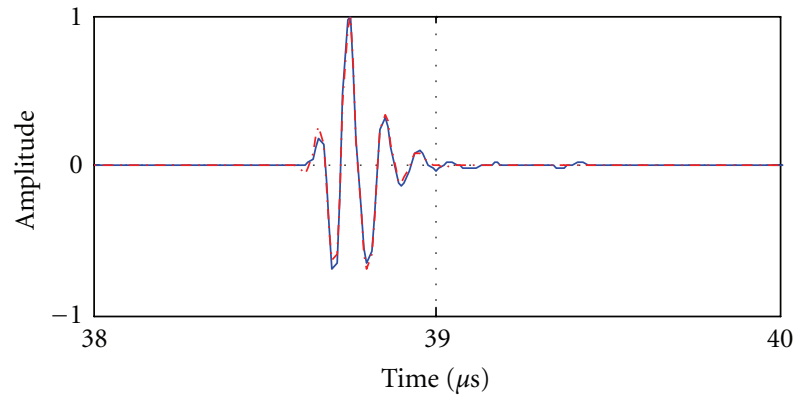

(b)

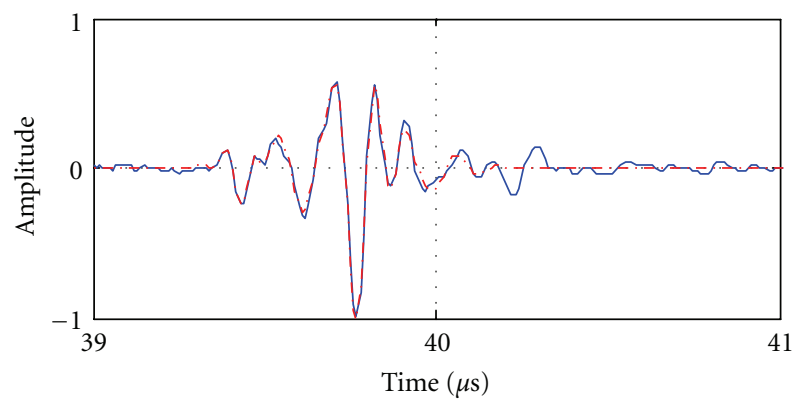

(d)

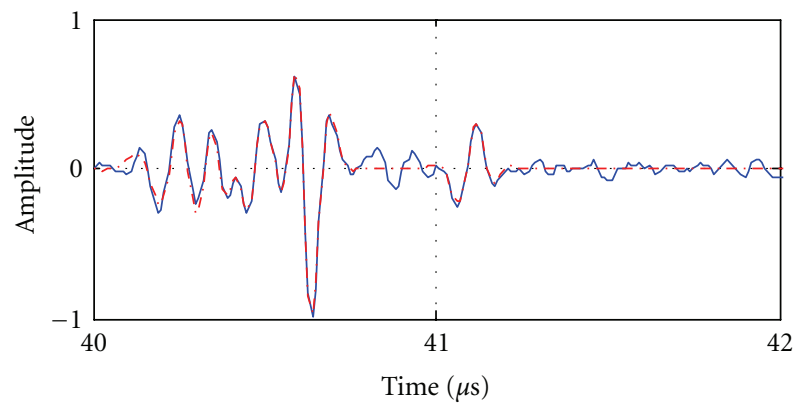

(f)

Figure 12: Experimental data of crack C (with normalized amplitudes) superimposed with the estimated chirplets. (a) Front surface reference signal superimposed with sum of 2 chirplets. (b) Experimental data (refracted angle 0) superimposed with sum of 2 chirplets. (c) Experimental data (refracted angle 30 at point a) superimposed with sum of 4 chirplets. (d) Experimental data (refracted angle 30 at point b) superimposed with sum of 4 chirplets. (e) Experimental data (refracted angle 45 at point a) superimposed with sum of 4 chirplets. (f) Experimental data (refracted angle 45 at point b) superimposed with sum of 4 chirplets.

TABLE 4: Estimated parameters of chirplets (block with $1 \mathrm{~mm} \mathrm{SDH).}$

\begin{tabular}{lcccc}
\hline & \multirow{2}{*}{ Chirplet parameters } & \multicolumn{3}{c}{ Refracted angle } \\
& & $0^{\circ}$ & $30^{\circ}$ & $45^{\circ}$ \\
\hline \multirow{3}{*}{$\begin{array}{l}\text { Spherically focused } \\
\text { transducer }\end{array}$} & Amplitude (m-Volt) & 42.5 & 29 & 16.01 \\
& TOA (us) & 76.62 & 82.6 & 89.39 \\
& Frequency (MHz) & 4.55 & 4.6 & 4.32 \\
\hline \multirow{3}{*}{ Planar transducer } & Amplitude (m-Volt) & 22.71 & 20.43 & 14.53 \\
& TOA (us) & 76.57 & 82.80 & 89.82 \\
& Frequency (MHz) & 4.48 & 4.67 & 4.81 \\
\hline
\end{tabular}

the transform order from (7) (i.e., -0.013 ) is used for a comparison. Our simulation shows that the optimal transform order for the band-limited echo is different compared with
TABLE 5: Estimated parameters of chirplets (block with $4 \mathrm{~mm} \mathrm{SDH}$ ).

\begin{tabular}{lcccc}
\hline & \multirow{2}{*}{ Chirplet parameters } & \multicolumn{3}{c}{ Refracted angle } \\
& & $0^{\circ}$ & $30^{\circ}$ & $45^{\circ}$ \\
\hline \multirow{3}{*}{$\begin{array}{l}\text { Spherically focused } \\
\text { transducer }\end{array}$} & Amplitude (m-Volt) & 87.75 & 59.34 & 32.61 \\
& Time of arrival (us) & 76.10 & 82.05 & 88.88 \\
& & & & \\
& Frequency (MHz) & 4.61 & 4.54 & 4.39 \\
\hline \multirow{3}{*}{ Planar transducer } & Amplitude (m-Volt) & 41.72 & 37.62 & 27.97 \\
& Time of arrival (us) & 76.11 & 82.36 & 89.42 \\
& Frequency (MHz) & 4.46 & 4.67 & 4.84 \\
\hline
\end{tabular}

the one for the LFM echo due to the impact of bandwidth factor in chirp echoes. 


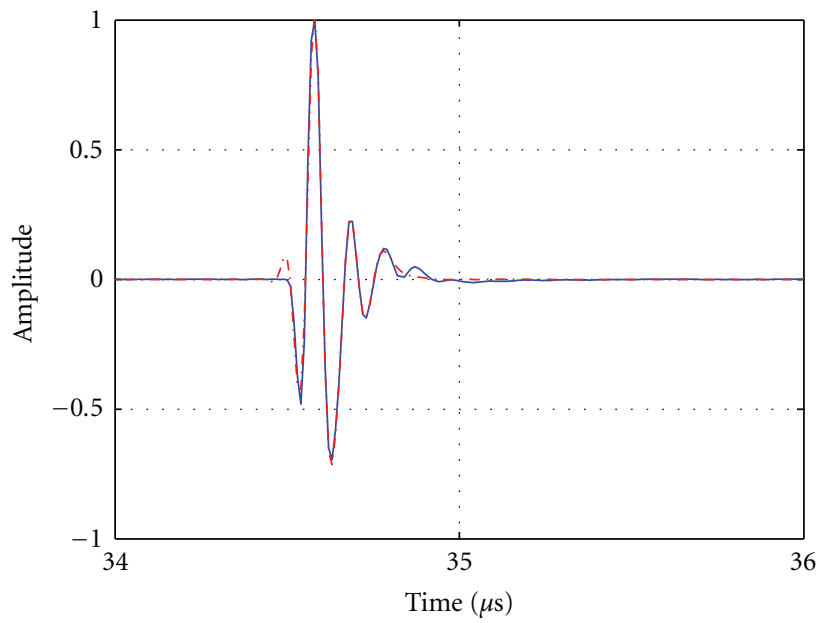

(a)

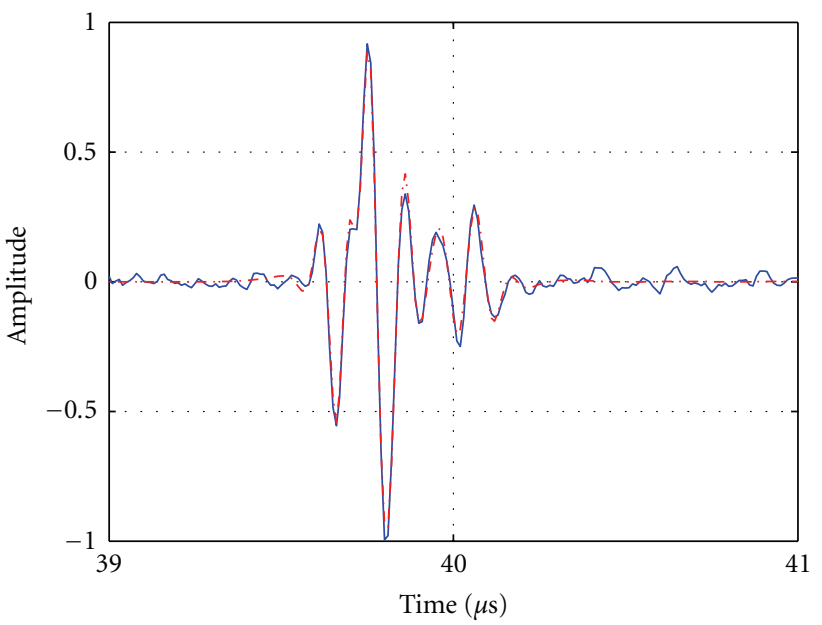

(c)

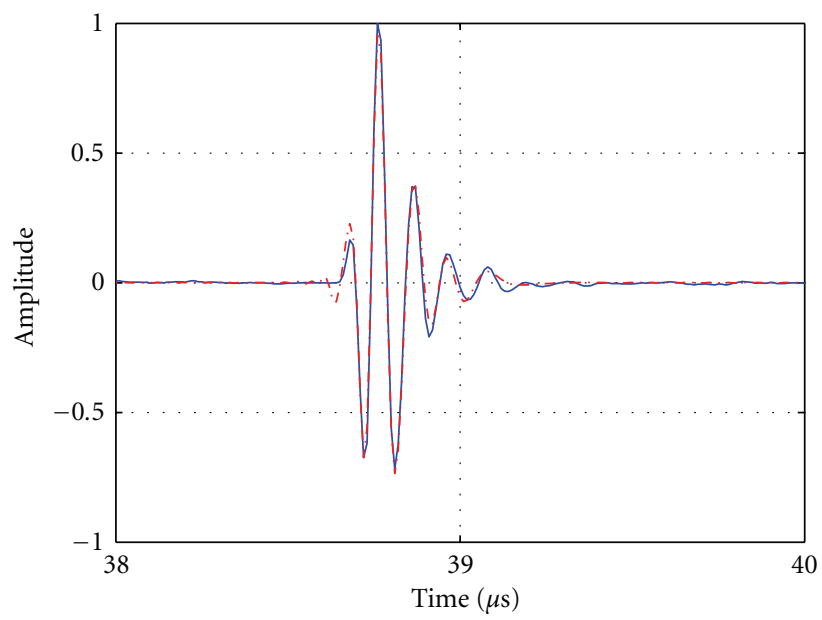

(b)

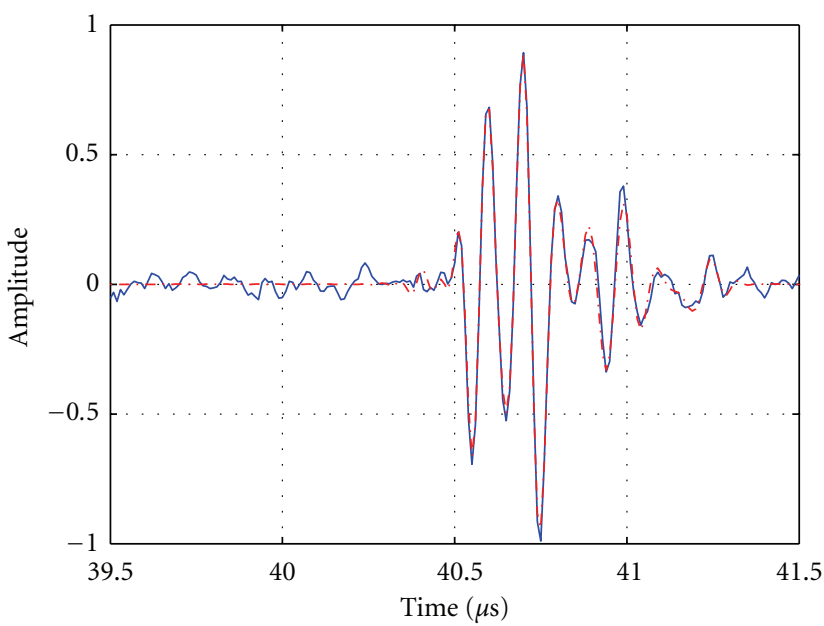

(d)

Figure 13: Experimental data of Crack D (with normalized amplitudes) superimposed with the estimated chirplets (depicted in dashed red line). (a) Front surface reference signal superimposed with sum of 2 chirplets. (b) Experimental data (refracted angle 0) superimposed with sum of 2 chirplets. (c) Experimental data (refracted angle 30) superimposed with sum of 4 chirplets. (d) Experimental data (refracted angle 45) superimposed with sum of 4 chirplets.

TABLE 6: Estimated parameters of chirplets (crack D).

\begin{tabular}{|c|c|c|c|}
\hline & TOA (us) & Center frequency $(\mathrm{MHz})$ & Amplitude (m-Volt) \\
\hline \multirow{2}{*}{ Reference signal } & 34.583 & 9.42 & 363.3 \\
\hline & 34.725 & 10.60 & 54.4 \\
\hline \multirow{2}{*}{ Refracted angle $0^{\circ}$} & 38.776 & 10.38 & 4.64 \\
\hline & 38.891 & 13.06 & 0.50 \\
\hline \multirow{4}{*}{ Refracted angle $30^{\circ}$} & 39.777 & 7.68 & 0.50 \\
\hline & 40.040 & 9.10 & 0.14 \\
\hline & 39.674 & 12.57 & 0.18 \\
\hline & 39.861 & 2.18 & 0.03 \\
\hline \multirow{4}{*}{ Refracted angle $45^{\circ}$} & 40.677 & 9.85 & 0.17 \\
\hline & 40.956 & 9.85 & 0.07 \\
\hline & 40.675 & 4.51 & 0.04 \\
\hline & 40.620 & 15.65 & 0.03 \\
\hline
\end{tabular}




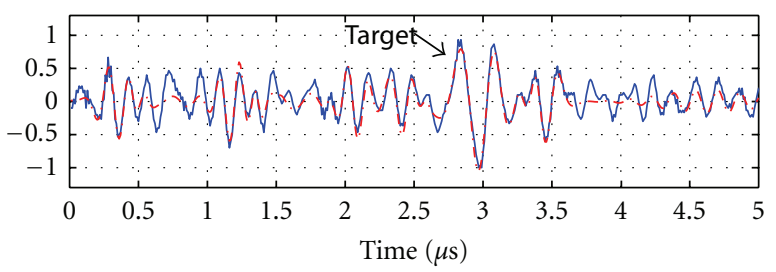

(a)

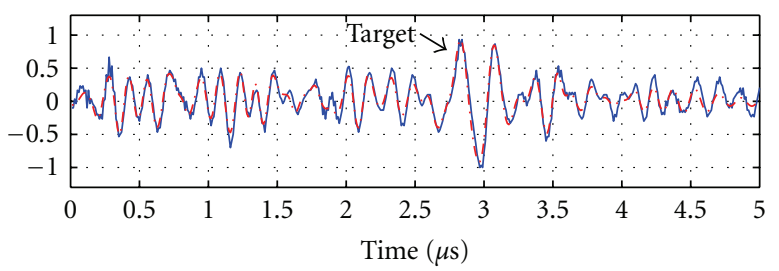

(b)

FIGURE 14: (a) Measured ultrasonic backscattered signal (blue) superimposed with the reconstructed signal consisting of 8 dominant chirplets (red). (b) Measured ultrasonic backscattered signal (blue) superimposed with the reconstructed signal consisting of 23 chirplets (red).

One can conclude that the compactness in the fractional Fourier transform of an ultrasonic echo can be used to track the optimal transform order. It is also important to point out that the optimal transform order is highly sensitive to a small change in the order. Therefore, using kurtosis becomes a practical approach to obtain the optimal FrFT order for ultrasonic signal analysis.

\section{FrFT Chirplet Signal Decomposition Algorithm}

The objective of FrFT-CSD is to decompose a highly convoluted ultrasonic signal, $s(t)$, into a series of signal components:

$$
s(t)=\sum_{j=1}^{N} f_{\Theta j}(t)+r(t),
$$

where $f_{\Theta j}(t)$ denotes the $j$ th fractional chirplet component and $r(t)$ denotes the residue of the decomposition process.

The steps involved in the iterative estimation of an experimental ultrasonic signal are

(1) initialize the iteration index $j=1$;

(2) obtain a windowed signal $s_{-}$win $(t)$ after applying a window, $w_{j}(t)$, in time domain;

$$
s_{\operatorname{win}(t)}=s(t) \times w_{j}(t)
$$

(3) determine the FrFT of the signal, $s_{-} w i n(t)$, $\operatorname{FrFT}^{\alpha}(x)_{s_{-} \operatorname{win}(t)}$, for different orders, $\alpha$;
(4) calculate kurtosis of $\operatorname{FrFT}^{\alpha}(x)_{s_{-} \text {win }(t)}$ for different orders, $\alpha$ :

$$
K(\alpha)=\frac{\mu_{4}\left(\operatorname{FrFT}^{\alpha}(x)_{s_{-} \text {win }(t)}\right)}{\left[\mu_{2}\left(\operatorname{FrFT}^{\alpha}(x)_{s_{-} \operatorname{win}(t)}\right)\right]^{2}} ;
$$

(5) estimate the optimal transform order, $\alpha_{\mathrm{opt}}$ :

$$
\alpha_{\mathrm{opt}}=\arg _{\alpha} \operatorname{MAX}(K(\alpha))
$$

$\alpha_{o p t}$ corresponds to the FrFT transform order where $K(\alpha)$ has the max value. In our study, a brute-force search is used to estimate the optimal transform order. The step size of searching is set to 0.005 . The computation load of calculating the kurtosis and searching for the optimal order is significant. Some researchers used the maximum peak in the transform domain as an alternative metric [17]. For ultrasonic signal decomposition, the optimal transform order is related to the chirp rate of the signal. The search range of transform order can be reduced by considering prior knowledge of ultrasonic transducer impulse response;

(6) apply FrFT with the estimated order $\alpha_{\text {opt }}$ to the signal $s(t)$ and obtain $\operatorname{FrFT}^{\alpha_{\mathrm{opt}}}(x)_{s(t)}$;

(7) obtain a windowed signal from $\operatorname{FrFT}^{\alpha_{\mathrm{opt}}}(x)_{s(t)}$ :

$$
\operatorname{FrFT}_{\text {win }}(x)=\operatorname{FrFT}^{\alpha_{\text {opt }}}(x)_{s(t)} \times \operatorname{win}_{j}(x),
$$

(8) apply the transformation order, $-\alpha_{\text {opt }}$, to the signal FrFT_win $(x)$, then reconstruct the $j$ th component by estimating parameters of the decomposed echo:

$$
f_{\Theta_{j}}(t)=\operatorname{FrFT}^{-\alpha_{\text {opt }}}(t)_{\text {FrFT_win }_{(x)}},
$$

the parameter estimation process here becomes a single-echo estimation problem. A Gaussian-Newton algorithm used in [23-25] is adopted in FrFT-CSD;

(9) obtain the residual signal by subtracting the estimated echo from the signal, $s(t)$, and use the residual signal for next echo estimation;

(10) calculate energy of residual signal $\left(E_{r}\right)$ and check convergence: ( $E_{\min }$ is predefined convergence condition) If $E_{r}<E_{\min }$, STOP; otherwise, go to step 2.

For further clarification, the flowchart of FrFT-CSD algorithm is shown in Figure 3. It is important to mention that two windowing steps are used in FrFT-CSD algorithm. One window is used in step 2 in order to isolate a dominant echo in time domain. It is inevitable to have an incomplete echo due to windowing process. A good strategy of choosing this window is to keep as much of echo information as possible. The other window is applied in step 7. For ultrasonic chirp echoes, the energy compactness of FrFT helps to reduce the window size centered on a desired peak in the transform domain. As shown in Figure 2, a chirplet is compressed to a great extent after the transform. An 
automatic windowing process is used to detect the valleys of the dominant echo. In the cases of heavily overlapping echoes and high noise levels (i.e., the cases of poor signalto-noise ratio), the performance of windowing method may be compromised. In this situation, a window with a predetermined size can be used to isolate desirable peaks.

\section{Simulation and Benchmark Study of FrFT-CSD}

To demonstrate the advantages of FrFT signal decomposition in ultrasonic signal processing, ultrasonic chirp echoes with three different overlapping scenarios are simulated, where chirp rate models the dispersive effect in ultrasonic testing of materials. Two slightly overlapped (about 20\% overlapped) echoes is simulated using the sampling frequency of $100 \mathrm{MHz}$. The parameters of these two echoes are

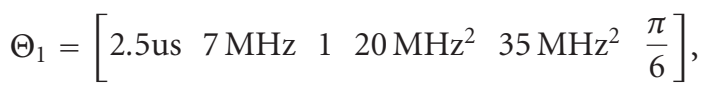

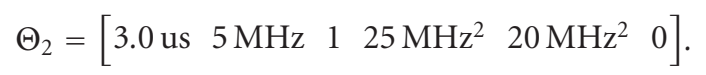

Figure 4 shows the simulated signal (in blue) superimposed with estimated echoes (in red). The estimated parameters perfectly match the parameters of simulation signal as compared in Table 1. One can conclude that the FrFT-CSD not only decomposes the signal efficiently, but also leads to precise parameter estimation results. A moderately overlapped (about 50\% overlapped) simulated signal consisting of two echoes is shown in Figure 5. For this simulated signal, Table 2 shows that the estimated parameters are accurate within a few percents.

Finally, Figure 6 and Table 3 show the simulated and estimated two heavily overlapped (about $70 \%$ overlapped) echoes. The decomposition results (Figure 6) and estimated parameters (Table 3 ) confirm the robustness and effectiveness of FrFT-CSD in echo estimation for ultrasonic signal analysis.

An experimental bat data is commonly used as a benchmark signal in time-frequency analysis. It is a 400sample data digitized $2.5 \mu$ s echolocation pulse emitted by a large brown bat with $7 \mu$ s sampling period. To evaluate the performance of FrFT-based signal decomposition algorithm, the bat data is utilized to demonstrate the effectiveness of algorithm.

Through the processing of FrFT-CSD, there are four main chirp-type signal components identified in the bat signal. The decomposed signals and their Wigner-Ville distribution (WVD) are shown in Figure 7. The reconstructed signal and its superimposed WVD are shown in Figure 8. The results in Figures 7 and 8 are consistent with the analysis results from other techniques in time-frequency analysis [26]. The FrFT-based signal decomposition algorithm not only reveals that the bat signal mainly contains four chirp stripes in time-frequency domain, but provides a highresolution time-frequency representation.

\section{Experimental Studies}

For experimental studies, two aluminum blocks with different size of side-drilled hole (SDH) are used [27]. One is with $1 \mathrm{~mm}$ diameter, another is $4 \mathrm{~mm}$ diameter. The experimental setting is shown in Figure 9. It can be seen that the water path is $50.8 \mathrm{~mm}$ and the depth of $\mathrm{SDH}$ is $25.4 \mathrm{~mm}$ (i.e., from the water-aluminum interface to the center of $\mathrm{SDH}$ ).

To provide a rigorous test, two $5 \mathrm{MHz}$ transducers are used to acquire ultrasonic data at normal or oblique refracted angles, $\theta$. One is planar transducer. Another is spherically focused transducer with $172.9 \mathrm{~mm}$ focal length.

To verify the experiment setup, the FrFT-CSD is utilized to analyze the ultrasonic data from the front surface of the specimen. The ultrasonic data superimposed with the estimated chirplet is shown in Figure 10.

It can be seen that the estimated time-of-arrival (TOA) of the front surface echo is $68.72 \mu \mathrm{s}$. In addition, from the experimental setting, the TOA can be calculated as

$$
\mathrm{TOA}=\frac{2 \times D}{v}
$$

where $D$ denotes the water distance, and in the case of incidence angle 0 this distance is $50.8 \mathrm{~mm}$. The round trip of ultrasound is twice of the water distance, $D$. The term $v$ denotes the velocity of ultrasound in medium: $v=$ $1.484 \mathrm{~mm} / \mu \mathrm{s}$ for water.

From (15), the theoretical value of TOA is $68.47 \mu \mathrm{s}$. The estimated TOA is in agreement (within $0.4 \%$ ) with the theoretical TOA.

Furthermore, the parameters of chirplet are strongly related to the crack size, location, and orientation. For example, the amplitude is a good indicator of crack size. In Tables 4 and 5, the estimated amplitude from a $4 \mathrm{~mm} \mathrm{SDH}$ is roughly twice of the estimated amplitude from a $1 \mathrm{~mm}$ SDH. In NDE applications, the estimated amplitude of a known-size crack could be used as a reference to estimate the size of crack. As shown in (15) and (16), the estimated TOA can be used to approximate the location of crack. In addition, different types of cracks could have different frequency variations. From $[8,26]$, the response of crack usually shows a downshift in the frequency compared with the responses of grains inside the material.

These results indicate that the estimated parameters from FrFT-CSD algorithm track with reasonable accuracy the physical parameters of experimental setup. Moreover, the FrFT-CSD algorithm provides more detailed information describing the reflected echoes such as phase, bandwidth factor and chirp rate that can be used for further analysis.

Another experiment is set up to evaluate disk-shaped cracks in a diffusion-bonded titanium alloy sample [28]. The ultrasonic data of these synthetic cracks are obtained at normal or oblique refracted angles, $\theta$ using a $10 \mathrm{MHz}$ planar transducer. The diameter of the transducer is $6.35 \mathrm{~mm}$. The water depth is $25.4 \mathrm{~mm}$. The surface of diffusion bond is $13 \mathrm{~mm}$ below the front surface of water/titanium alloy interface. Two different sizes of cracks are made with the diameter $0.762 \mathrm{~mm}$ (i.e., crack D) and the diameter $1.905 \mathrm{~mm}$ (i.e., crack C). For crack $\mathrm{C}$, the responded ultrasonic data is 
TABLE 7: Estimated parameters of chirplets ( $\operatorname{crack} \mathrm{C})$.

\begin{tabular}{|c|c|c|c|}
\hline & TOA (us) & Center frequency $(\mathrm{MHz})$ & Amplitude (m-Volt) \\
\hline \multirow{2}{*}{ Reference signal } & 34.583 & 9.42 & 363.3 \\
\hline & 34.725 & 10.60 & 54.4 \\
\hline \multirow{2}{*}{ Refracted angle $0^{\circ}$} & 38.754 & 9.78 & 14.48 \\
\hline & 38.863 & 12.93 & 1.86 \\
\hline \multirow{4}{*}{ Refracted angle $30^{\circ}$ (point a) } & 39.784 & 11.02 & 0.58 \\
\hline & 40.029 & 6.06 & 0.19 \\
\hline & 40.560 & 7.68 & 0.13 \\
\hline & 40.122 & 10.63 & 0.06 \\
\hline \multirow{4}{*}{ Refracted angle $45^{\circ}$ (point a) } & 40.825 & 9.88 & 0.14 \\
\hline & 41.157 & 9.92 & 0.07 \\
\hline & 40.795 & 15.64 & 0.04 \\
\hline & 41.658 & 6.87 & 0.05 \\
\hline \multirow{4}{*}{ Refracted angle $30^{\circ}$ (point b) } & 39.757 & 7.78 & 0.49 \\
\hline & 39.536 & 5.32 & 0.11 \\
\hline & 39.905 & 4.63 & 0.10 \\
\hline & 39.426 & 11.13 & 0.10 \\
\hline \multirow{4}{*}{ Refracted angle $45^{\circ}$ (point b) } & 40.632 & 9.09 & 0.21 \\
\hline & 40.270 & 9.65 & 0.07 \\
\hline & 40.468 & 3.40 & 0.16 \\
\hline & 41.100 & 7.97 & 0.07 \\
\hline
\end{tabular}

TABLE 8: Estimated parameters of the 8 dominant chirplets for ultrasonic experimental data.

\begin{tabular}{lcccccc}
\hline & $\tau$ (us) & $f_{c}(\mathrm{MHz})$ & $\beta$ & $\alpha_{1}(\mathrm{MHz})^{2}$ & $\alpha_{2}(\mathrm{MHz})^{2}$ & $\theta(\mathrm{Rad})$ \\
\hline Echo 1 & 2.95 & 3.87 & 1.06 & 20.16 & 5.17 & -41.06 \\
Echo 2 & 3.47 & 5.53 & 0.63 & 0.54 & 37.45 & 2.80 \\
Echo 3 & 0.33 & 6.57 & 0.54 & 27.14 & 30.17 & 1.76 \\
Echo 4 & 1.18 & 7.24 & 0.53 & 39.13 & -15.50 & 3.71 \\
Echo 5 & 2.08 & 6.66 & 0.47 & 62.91 & 60.24 & 2.75 \\
Echo 6 & 2.40 & 6.00 & 0.18 & 4.75 & -0.18 & -2.47 \\
Echo 7 & 4.64 & 6.23 & 0.12 & 0.73 & -0.04 \\
Echo 8 & 1.49 & 3.97 & & & -6.64 \\
\hline
\end{tabular}

recorded from the two edges of the crack, which are marked as point $\mathrm{a}$ and point $\mathrm{b}$. The thickness of both disk-shaped cracks is $0.089 \mathrm{~mm}$. Figure 11 shows the experiment setup for the alloy sample [28].

From Figure 11, the TOA of crack at refracted angle $\theta$ is calculated as follows:

$$
\mathrm{TOA}_{\theta}=\mathrm{TOA}_{\mathrm{ref}}+\frac{2 \times D / \cos \theta}{v}
$$

where $\mathrm{TOA}_{\text {ref }}$ denotes the estimated TOA of reference signal (i.e., $34.58 \mu \mathrm{s}$ from Tables 6 and 7). The round trip of ultrasound inside titanium from the front surface to the diffusion bound is $2 \times D / \cos \theta$, where $D$ denotes the depth of diffusion bond, which is $13 \mathrm{~mm} ; \theta$ denotes the refracted angle and $v$ denotes the velocity of ultrasound in medium: $v=6.2 \mathrm{~mm} / \mu$ s for titanium. Therefore, $\operatorname{TOA}_{\theta}$ at the angle $0^{\circ}$ is $38.777 \mu \mathrm{s} . \mathrm{TOA}_{\theta}$ at the angle $30^{\circ}$ is $39.425 \mu \mathrm{s}$. At the angle $45^{\circ}, \mathrm{TOA}_{\theta}$ is $40.514 \mu \mathrm{s}$.

From Tables 6 and 7, it can be seen that the estimated $\mathrm{TOA}_{\theta}$ at angle $0^{\circ}$ is $38.776 \mu \mathrm{s}$ and $38.754 \mu \mathrm{s}$. Taking the thickness of the cracks $(0.089 \mathrm{~mm})$ into consideration, it can be asserted that the estimated TOAs at incident angle $0^{\circ}$ are in good agreement with experimental measurements. Experimental signals of crack C and crack D (with normalized amplitudes) superimposed with the estimated chirplets (depicted in dashed line and red color) are shown in Figures 12 and 13. It also can be seen that the front surface reference signal and the experimental data obtained at angle $0^{\circ}$ are well reconstructed by the FrFT-CSD algorithm (see Figures 12(a), 12(b), 13(a) and 13(b)). Nevertheless, with the increase of refracted angle, more chirplets needed to decompose the experimental data (see the refracted angle 30 and 45 degree 
cases). In addition, Tables 6 and 7 show that the signal energy is more evenly distributed to estimated chirplets in the high refracted angle cases. This spreading of signal might be caused by geometrical effect of the beam profile of the planner transducer and corners/edges of disk-shaped crack.

To further evaluate the performance of FrFT-based signal decomposition algorithm, experimental ultrasonic microstructure scattering signals are utilized to demonstrate the effectiveness of the algorithm. The experimental signal is acquired from a steel block with an embedded defect using a $5 \mathrm{MHz}$ transducer and sampling rate of $100 \mathrm{MHz}$. The acquired experimental data superimposed with the reconstructed signal consisting of 8 dominant chirplets are shown in Figure 14(a). The estimated parameters of dominant chirplets are listed in Table 8. It can be seen that the 8 dominant chirplets not only provide a sparse representation of experimental data, but successfully detect the embedded defect.

To improve the accuracy of signal reconstruction, FrFTCSD could be used iteratively to decompose the signal further. A reconstructed signal using 23 chirplets is shown in Figure 14(b). The comparison between the experimental signal and the reconstructed signals clearly demonstrates that the FrFT-CSD is highly effective in ultrasonic signal decomposition.

\section{Conclusion}

In this paper, fractional Fourier transform is studied for ultrasonic signal processing. Simulation study reveals the link among kurtosis, the transform order, and the parameters of each decomposed components. Benchmark and experimental data sets are utilized to test the FrFT-based chirplet signal decomposition algorithm. Signal decomposition and parameter estimation results show that fractional Fourier transform can successfully assist signal decomposition algorithm by identifying the dominant echo in successive estimation iteration. Parameter estimation is further performed based on the echo isolation. The FrFT-CSD algorithm could have a broad range of applications in signal analysis including target detection and pattern recognition.

\section{Acknowledgments}

The authors wish to thank Curtis Condon, Ken White, and Al Feng of the Beckman Institute of the University of Illinois for the bat data and for permission to use it in the study.

\section{References}

[1] S. Mallat, A Wavelet tour of Signal Processing: The Sparse Way, Academic Press, 2008.

[2] I. Daubechies, "The wavelet transform, time-frequency localization and signal analysis," IEEE Transactions on Information Theory, vol. 36, no. 5, pp. 961-1005, 1990.

[3] S. Mann and S. Haykin, "The chirplet transform: physical considerations," IEEE Transactions on Signal Processing, vol. 43, no. 11, pp. 2745-2761, 1995.
[4] G. Cardoso and J. Saniie, "Ultrasonic data compression via parameter estimation," IEEE Transactions on Ultrasonics, Ferroelectrics, and Frequency Control, vol. 52, no. 2, pp. 313325, 2005.

[5] R. Tao, Y. L. Li, and Y. Wang, "Short-time fractional fourier transform and its applications," IEEE Transactions on Signal Processing, vol. 58, no. 5, pp. 2568-2580, 2010.

[6] S. Zhang, M. Xing, R. Guo, L. Zhang, and Z. Bao, "Interference suppression algorithm for SAR based on time frequency domain," IEEE Transaction on Geoscience and Remote Sensing, vol. 49, no. 10, pp. 3765-3779, 2011.

[7] E. Oruklu and J. Saniie, "Ultrasonic flaw detection using discrete wavelet transform for NDE applications," in Proceedings of IEEE Ultrasonics Symposium, pp. 1054-1057, August 2004.

[8] Y. Lu, R. Demirli, G. Cardoso, and J. Saniie, "A successive parameter estimation algorithm for chirplet signal decomposition," IEEE Transactions on Ultrasonics, Ferroelectrics, and Frequency Control, vol. 53, no. 11, pp. 2121-2131, 2006.

[9] S. C. Pel and J. J. Ding, "Closed-form discrete fractional and affine fourier transforms," IEEE Transactions on Signal Processing, vol. 48, no. 5, pp. 1338-1353, 2000.

[10] L. B. Almeida, "Fractional fourier transform and timefrequency representations," IEEE Transactions on Signal Processing, vol. 42, no. 11, pp. 3084-3091, 1994.

[11] C. Candan, M. Alper Kutay, and H. M. Ozaktas, "The discrete fractional fourier transform," IEEE Transactions on Signal Processing, vol. 48, no. 5, pp. 1329-1337, 2000.

[12] A. S. Amein and J. J. Soraghan, "The fractional Fourier transform and its application to High resolution SAR imaging," in Proceedings of IEEE International Geoscience and Remote Sensing Symposium (IGARSS '07), pp. 5174-5177, June 2007.

[13] M. Barbu, E. J. Kaminsky, and R. E. Trahan, "Fractional fourier transform for sonar signal processing," in Proceedings of MTS/IEEE OCEANS, vol. 2, pp. 1630-1635, September 2005.

[14] I. S. Yetik and A. Nehorai, "Beamforming using the fractional fourier transform," IEEE Transactions on Signal Processing, vol. 51, no. 6, pp. 1663-1668, 2003.

[15] S. Karako-Eilon, A. Yeredor, and D. Mendlovic, "Blind source separation based on the fractional Fourier transform," in Proceedings of the 4th International Symposium on Independent Component Analysis and Blind Signal Separation, pp. 615-620, 2003.

[16] A. T. Catherall and D. P. Williams, "High resolution spectrograms using a component optimized short-term fractional Fourier transform," Signal Processing, vol. 90, no. 5, pp. 15911596, 2010.

[17] M. Bennett, S. McLaughlin, T. Anderson, and N. McDicken, "Filtering of chirped ultrasound echo signals with the fractional fourier transform," in Proceedings of IEEE Ultrasonics Symposium, pp. 2036-2040, August 2004.

[18] L. Stanković, T. Alieva, and M. J. Bastiaans, "Time-frequency signal analysis based on the windowed fractional Fourier transform," Signal Processing, vol. 83, no. 11, pp. 2459-2468, 2003.

[19] Y. Lu, A. Kasaeifard, E. Oruklu, and J. Saniie, "Performance evaluation of fractional Fourier transform(FrFT) for timefrequency analysis of ultrasonic signals in NDE applications," in Proceedings of IEEE International Ultrasonics Symposium (IUS '10), pp. 2028-2031, October 2010.

[20] F. Millioz and N. Martin, "Circularity of the STFT and spectral kurtosis for time-frequency segmentation in Gaussian 
environment," IEEE Transactions on Signal Processing, vol. 59, no. 2, pp. 515-524, 2011.

[21] R. Merletti, A. Gulisashvili, and L. R. Lo Conte, "Estimation of shape characteristics of surface muscle signal spectra from time domain data," IEEE Transactions on Biomedical Engineering, vol. 42, no. 8, pp. 769-776, 1995.

[22] Y. Lu, E. Oruklu, and J. Saniie, "Analysis of Fractional Fouriter transform for ultrasonic NDE applications," in Proceedings of IEEE Ultrasonic Symposium, Orlando, Fla, USA, October 2011.

[23] R. Demirli and J. Saniie, "Model-based estimation of ultrasonic echoes part I: analysis and algorithms," IEEE Transactions on Ultrasonics, Ferroelectrics, and Frequency Control, vol. 48, no. 3, pp. 787-802, 2001.

[24] R. Demirli and J. Saniie, "Model-based estimation of ultrasonic echoes part II: nondestructive evaluation applications," IEEE Transactions on Ultrasonics, Ferroelectrics, and Frequency Control, vol. 48, no. 3, pp. 803-811, 2001.

[25] R. Demirli and J. Saniie, "Model based time-frequency estimation of ultrasonic echoes for NDE applications," in Proceedings of IEEE Ultasonics Symposium, pp. 785-788, October 2000.

[26] Y. Lu, E. Oruklu, and J. Saniie, "Ultrasonic chirplet signal decomposition for defect evaluation and pattern recognition," in Proceedings of IEEE International Ultrasonics Symposium (IUS '09), ita, September 2009.

[27] Ultrasonic Benchmark Data, World Federation of NDE, 2004, http://www.wfndec.org/.

[28] Ultrasonic Benchmark Data, World Federation of NDE, 2005, http://www.wfndec.org/. 

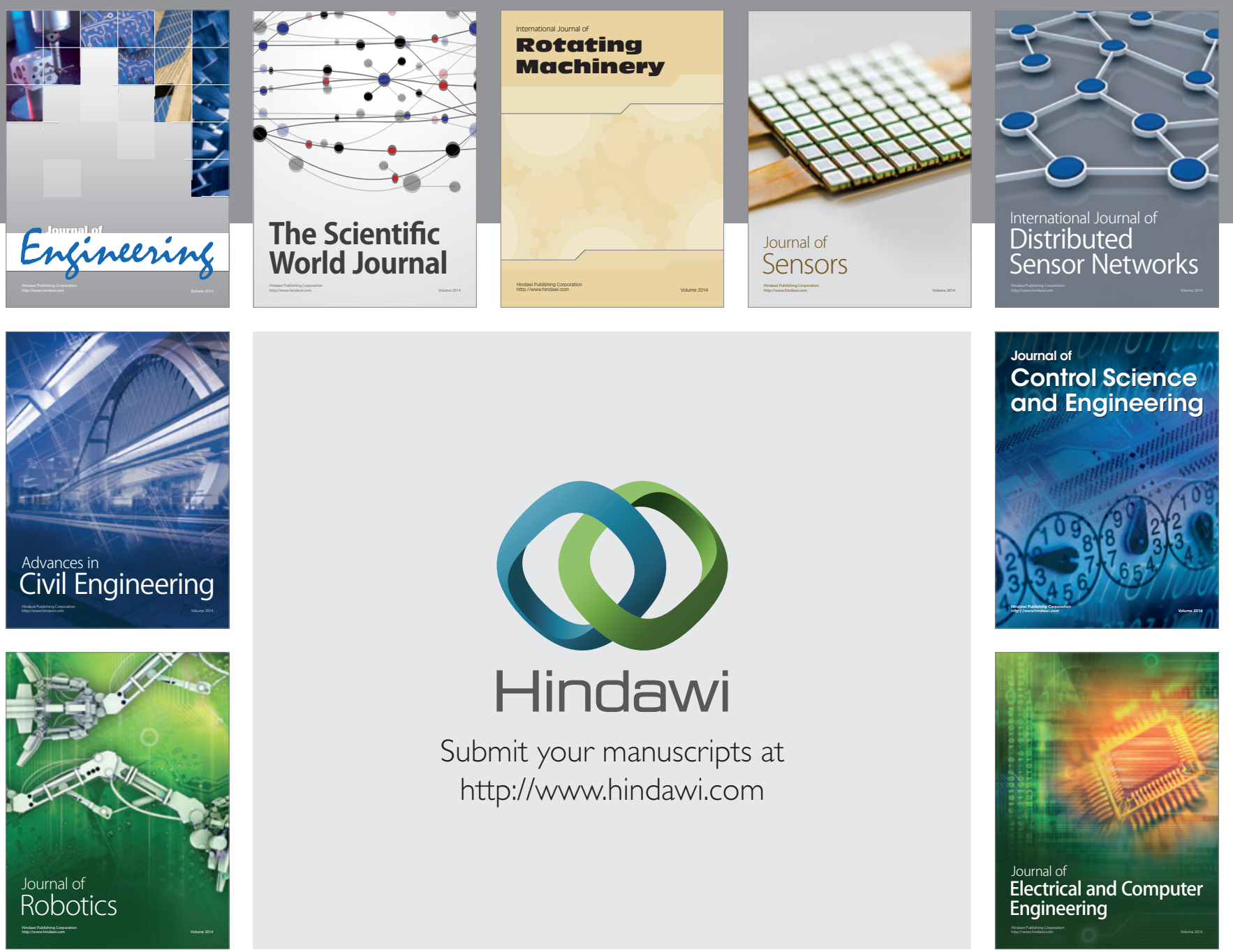

Submit your manuscripts at

http://www.hindawi.com
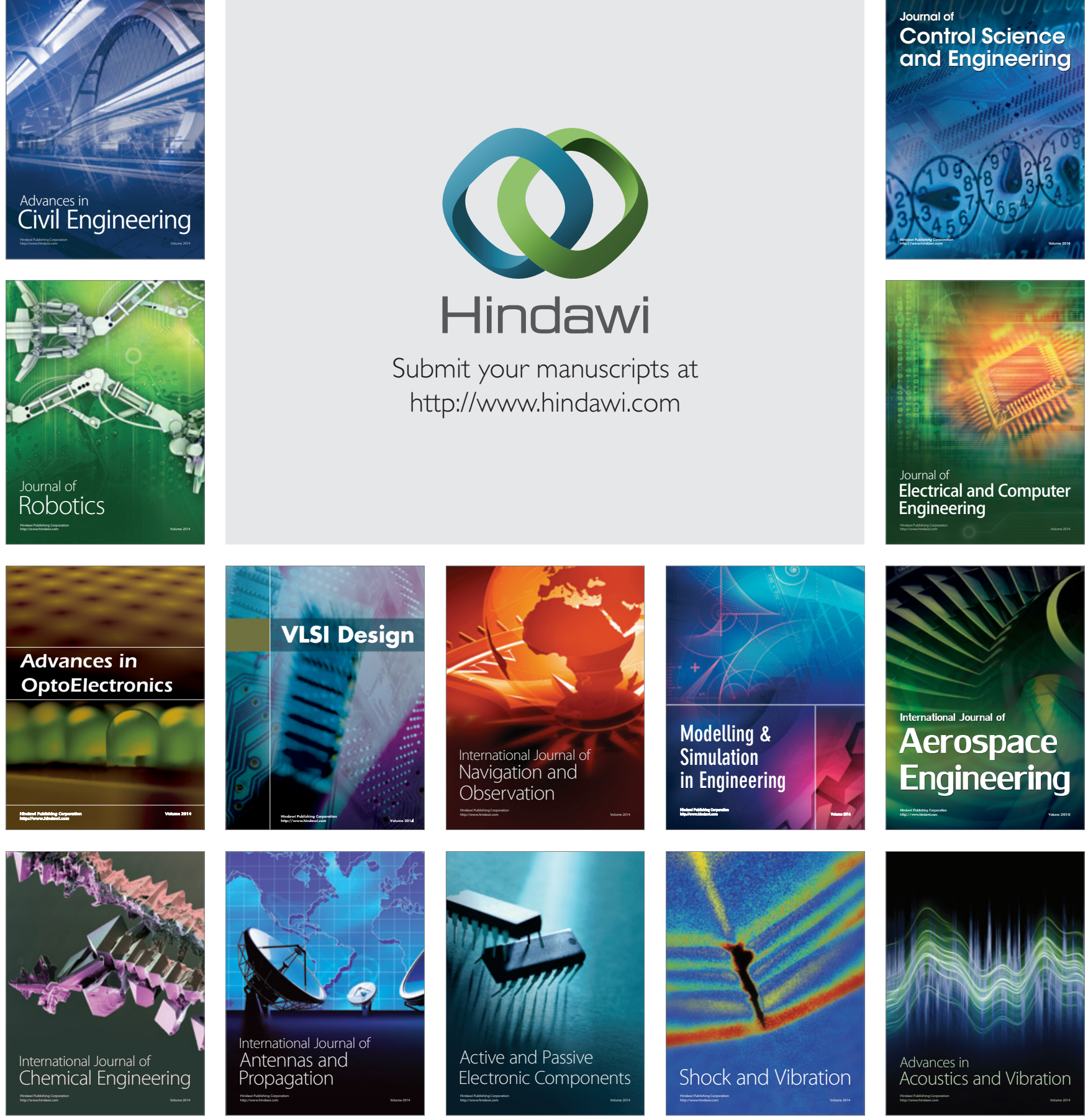